\title{
Modelo lógico-ideal para o estágio curricular supervisionado: a educação pelo trabalho na formação Odontológica
}

Graciela Soares Fonsêca*; Simone Rennó Junqueira**; Maria Ercília de Araújo***; Carlos Botazzo****

\author{
* Aluna de Doutorado do Programa de pós-graduação em \\ Ciências Odontológicas com área de concentração em \\ Odontologia Social da Faculdade de Odontologia da \\ Universidade de São Paulo. \\ ** Professora do Departamento de Odontologia Social da \\ Faculdade de Odontologia da Universidade de São \\ Paulo. \\ *** Professora do Departamento de Odontologia Social da \\ Faculdade de Odontologia da Universidade de São \\ Paulo. \\ **** Professor do Departamento de Prática de Saúde Pública \\ da Faculdade de Saúde Pública da Universidade de São \\ Paulo.
}

\section{RESUMO}

O estudo buscou elaborar um modelo lógico de Estágio Curricular Supervisionado (ECS) para os cursos de graduação em odontologia. Trata-se de um estudo transversal que levantou todos os resumos e artigos presentes nas revistas da Associação Brasileira de Ensino Odontológico (ABENO), publicados entre 2003 e 2012. Foram incluídos artigos que continham a palavra 'estágio' em seus títulos, sendo analisados 23 deles. As variáveis estudadas foram: ano de publicação, local de realização, duração, atividades desenvolvidas, contribuições para a formação, papel dos profissionais dos serviços e limites encontrados. Os dados referentes às três primeiras variáveis foram digitados em planilha do Microsoft Office Excel 2007® e analisados mediante distribuição de frequência. As demais variáveis foram trabalhadas com um enfoque qualitativo. A maioria dos trabalhos foi publicada nos anos de 2012 (13,04\%), 2011 (17,39\%) e 2010 (17,39\%). Os estágios eram realizados no âmbito do Sistema Único de Saúde (SUS) $(73,91 \%)$, sendo 39,13\% desenvolvidos restritamente, no nível básico. A maioria deles durava um semestre $(69,56 \%)$. Os ECS contribuem para uma formação coerente com o mundo do trabalho, oportuniza a vivência do trabalho em equipe e desperta os alunos para a atuação futura na saúde pública. $\mathrm{O}$ modelo lógico proposto ressalta a importância da inserção longitudinal nos serviços de saúde, prioriza a compreensão da organização dos serviços de saúde de forma integral e o entendimento do SUS no seu cotidiano, com seus limites e desafios.

Descritores: Odontologia. Educação Superior. Recursos Humanos. Saúde Bucal. 


\section{INTRODUÇÃO}

Os Estágios Curriculares Supervisionados (ECS) são constituídos de maneira institucionalizada, garantidos nas estruturas curriculares, visando proporcionar a formação por meio do trabalho nas mais diferentes áreas profissionais.

A legislação federal que dispõe sobre o estágio de estudantes o define como um "ato educativo escolar supervisionado, desenvolvido no ambiente de trabalho, que visa à preparação para o trabalho produtivo de educandos que estejam frequentando o ensino regular em instituições de educação superior". Ainda segundo a mesma legislação, "visa ao aprendizado de competências próprias da atividade profissional e a contextualização curricular, objetivando o desenvolvimento do educando para a vida cidadã e para o trabalho" .

Estágio pode ser conceituado, ainda, como a prática de campo que proporciona aos estudantes a transição do mundo acadêmico para o mundo do trabalho. Ele se conforma por uma série de atividades desenvolvidas pelos estudantes compondo o seu processo formativo $^{2}$.

Por serem previstos no projeto pedagógico do curso de graduação são chamados de obrigatórios. Têm carga horária pré-estabelecida, sem a qual não há aprovação. Além disso, deverão ser supervisionados pelo professor orientador da Instituição de Ensino Superior (IES) e por um supervisor da parte concedente, ou seja, por um trabalhador dos serviços de saúde ${ }^{1}$.

Acredita-se que o modelo de estágio ideal deve ter seus objetivos definidos também em função das deman- das dos serviços de saúde, com ações voltadas para o serviço, numa visão integral e de forma interdisciplinar, não permitindo que os objetivos das disciplinas criem uma pseudo-realidade. Além disso, idealiza-se uma inserção longitudinal em que o estudante crie vínculos com os serviços de saúde, de forma a sentir-se corresponsável pelas suas práticas.

"A integração entre uma instituição de ensino superior e outra, de serviços de saúde, acontece de fato quando existe, para ambas, uma intencionalidade complementar $e$ convergente, com objetivos comuns e peculiares, capazes de permitir a constituição de espaços pedagógicos com vivências e experimentações que possibilitem, para ambas, ganhos reais. A cada momento, a própria realidade deve ser o objeto do aprendizado, havendo lugar para o previsto e o imprevisto, o conhecido e o desconhecido, o já experimentado e o novo. $O$ aprendizado ocorrerá por meio de novas práticas, também pedagógicas, a partir de vivências e experimentações com a garantia do lugar de sujeito para professores, profissionais dos serviços, alunos $e$ usuários. A nosso ver, estes são, por excelência, os cenários (pedagógicos) ideais ao desenvolvimento dos estágios curriculares supervisionados".

Nos primeiros anos do século XXI, todos os cursos de graduação na área saúde estavam se mobilizando para produzir modificações no interior dos seus processos de formação. Iniciaram-se 
mudanças profundas no pensar a formação em saúde que se imprimiram nas Diretrizes Curriculares Nacionais (DCN) elaboradas para os diversos cursos. Em suma, elas legislaram a aproximação das IES com o Sistema Único de Saúde (SUS), possibilitaram uma base comum de orientação para a reestruturação curricular nas várias profissões da saúde, compartilhando competências entre os cursos e orientações como: articulação com o SUS, diversificação dos cenários de prática e a relevância da aprendizagem participativa na organização curricular e se constituíram na base para que o Ministério da Saúde desenvolvesse - muitas vezes em parceria com o Ministério da Educação iniciativas políticas para estimular a reorientação da formação em saúde ${ }^{4}$.

No caso da odontologia, as DCN preveem que o processo de formação dos profissionais garanta o desenvolvimento de estágio curricular, sob supervisão docente. O ECS deve ser desenvolvido de forma articulada e com complexidade crescente ao longo do processo de formação. A carga horária mínima do ECS deverá atingir 20\% da carga horária total do curso de graduação ${ }^{5}$.

As DCN colocam, ainda, que os profissionais de saúde bucal necessitam aprender a aprender de forma a torná-los corresponsáveis pela sua formação e pelo treinamento das futuras gerações profissionais. Assim, deve ser garantido o benefício mútuo entre os futuros profissionais e os profissionais dos serviços ${ }^{5}$.

A Associação Brasileira de Ensino Odontológico (ABENO), no documento intitulado "Diretrizes da ABENO para a definição do ECS nos cursos de odontologia" estabeleceu, com base nas
DCN, as diretrizes norteadoras dos estágios $^{6}$.

$\mathrm{O}$ documento afirma que "O estágio supervisionado é o instrumento de integração e conhecimento do aluno com a realidade social e econômica de sua região e do trabalho de sua área. Ele deve também ser entendido como o atendimento integral ao paciente que o aluno de Odontologia presta à comunidade, intra e extramuros. $\mathrm{O}$ aluno pode cumpri-lo em atendimentos multidisciplinares e em serviços assistenciais públicos e privados"6.

O ECS deve fomentar a relação das IES com os serviços e ampliar as relações da universidade com a sociedade. Como ferramenta pedagógica, deve proporcionar ao futuro profissional a experienciação da realidade social, incluindo as práticas políticas em saúde pública e a realidade do mercado de trabalho, possibilitando ao aluno ser um agente transformador dessas realidades ${ }^{6}$.

Ao analisar esse documento, Werneck et al. ${ }^{3}$ teceram algumas críticas, dentre elas a variedade de experiências que o documento denominou de ECS, o que impossibilita a definição dos critérios adotados para classificá-los dessa maneira.

"As citações são de tal forma genéricas que se pode supor, inclusive, que as instituições de ensino poderiam simplesmente inserir qualquer atividade de atendimento ao público na categoria estágio, ferindo frontalmente a dimensão transformadora e inovadora que gerou o estabelecimento das DCN e do documento da própria ABENO"3. 
Para se configurar como ECS, a atividade deve ser obrigatória, desenvolvida fora dos muros da IES, de forma integrada aos serviços de saúde. Dessa maneira, viabiliza-se a vivência do futuro profissional com o quadro epidemiológico presente, sua determinação social e cultural, bem como a compreensão do seu verdadeiro papel e dos limites da sua atuação profissional. Além disso, é despertada no futuro cirurgião-dentista a noção do papel e do compromisso com o sistema de saúde vigente e com a população ${ }^{3}$.

A partir desse referencial teórico, foi possível esquematizar um quadroresumo (Figura 1) com as características essenciais que definem uma atividade curricular como estágio.

\begin{tabular}{|c|}
\hline $\begin{array}{c}\text { Características essenciais dos ECS } \\
\text { Regulamentados no Projeto Pedagógico do } \\
\text { curso }\end{array}$ \\
\hline Desenvolvidos fora dos muros da IES \\
\hline Integrados aos serviços de saúde \\
\hline Obrigatórios para a conclusão do curso de \\
graduação
\end{tabular}

Além disso, é imprescindível que os ECS se utilizem de características de outros modelos de inserção de alunos nos serviços de saúde, desenvolvidos como instrumentos de reorientação da formação, com intuito de garantir o sucesso das ações realizadas. Nesse contexto, o Programa de Educação pelo Trabalho para Saúde (PET-Saúde), mesmo tendo sido uma estratégia temporária, com sua proposta diferenciada e inovadora, des- ponta como um modelo mais próximo do ideal $^{7-9}$.

Considerando-se essas premissas, esse estudo buscou revelar a conformação dos estágios pelo território brasileiro, baseado em publicações de um dos periódicos mais relevantes para o tema, em um período determinado, no sentido de subsidiar a criação de um modelo lógico/ideal de ECS para os cursos de graduação em odontologia.

\section{METODOLOGIA}

Trata-se de estudo transversal que analisou todos os resumos e artigos presentes nas revistas da ABENO, publicados posteriormente à data de divulgação das DCN (2003-2012). O modelo lógico é a ideia que operacionaliza um objeto-modelo, que é uma situação real ou suposta como real ${ }^{10}$.

Para serem incluídos no estudo, os trabalhos deveriam conter a palavra 'estágio' em seus títulos. Após o levantamento inicial, procedeu-se uma análise do texto para verificar se, realmente, tratava-se de ECS. Nessa fase, eliminaram-se 31 trabalhos que, apesar de trazerem a palavra "estágio" no título, abordavam experiências disciplinares pontuais de interação com os serviços de saúde sem configurar-se, verdadeiramente, como um ECS ou, ainda, tratavam de outros temas como metodologias ativas de aprendizagem, expectativa dos estudantes sobre futuros estágios $\mathrm{e}$ estágios extracurriculares, dentre outros. Além disso, alguns resumos não traziam informações suficientes para serem analisados e outros eram repetidos, motivos pelos quais os estudos também 
foram descartados. Por fim, foram analisados 23 trabalhos.

As variáveis estudadas foram: ano de publicação, local de realização, duração, atividades desenvolvidas, contribuições para a formação, papel dos profissionais dos serviços e limites encontrados.

Os dados referentes às três primeiras variáveis foram digitados em planilha do Microsoft Office Excel 2007® e analisados mediante distribuição de frequência. As demais variáveis foram trabalhadas de modo qualitativo, por intermédio da análise de conteúdo temática de Bardin ${ }^{11}$.

\section{RESULTADOS E DISCUSSÃO}

\section{Sobre a sistematização dos dados}

Com relação ao ano de publicação, a maioria foi publicada nos anos de 2012 $(13,04 \%), \quad 2011 \quad(17,39 \%)$ e 2010 $(17,39 \%)$. Ainda que as IES tenham incluído práticas de ECS segundo o referencial proposto nas DCN logo após ou até mesmo antes delas serem instituídas, verificou-se que avaliações começaram a ser disponibilizadas apenas uma década depois.

Os estágios eram realizados no âmbito do SUS $(73,91 \%)$, sendo a maioria desenvolvidos, restritamente, no nível básico (39,13\%). Alguns trabalhos relataram associação dos níveis básicos e secundário $(4,34 \%)$ e até dos três níveis (26,08\%). Um dos estágios era desenvolvido somente no nível terciário $(4,34 \%)$.

Além disso, houve relato de associação das Unidades Básicas de Saúde (UBS) com as clínicas da faculdade $(4,34 \%)$, relatos de estágios desenvolvidos em hospitais privados $(8,69 \%)$, em escolas
$(8,69 \%)$ e em equipamentos sociais, como creches e abrigos para idosos (4,34\%).

A maioria dos estágios durava um semestre $(69,56 \%)$, mas havia os que tinham duração de um $(13,04 \%)$ ou dois $(13,04 \%)$ anos. Um trabalho relatou que o estágio perdurava durante os cinco anos do curso, no entanto, com a análise do resumo, ficou evidente que havia, ligadas à disciplina de estágio propriamente dito, disciplinas que promoviam a inserção dos alunos nos serviços de forma pontual.

A análise permitiu verificar que houve uma mudança positiva, ao longo dos anos, em relação à conformação dos ECS. Os trabalhos publicados a partir de 2010 trazem uma série de elementos importantes para avançar no modelo quando comparado aos trabalhos publicados anteriormente, o que favoreceu a reflexão sobre outras questões que serão discutidas a seguir.

Os trabalhos apontam o desenvolvimento de atividades de territorialização, visitas domiciliares, levantamentos epidemiológicos, acompanhamento de toda a equipe de saúde, atividades educativas em equipamentos sociais ligados à UBS e atendimento clínico, além da articulação das atividades práticas com discussões teóricas no âmbito das IES ${ }^{12-15}$.

Quando o estágio envolve também os níveis secundários e terciários, há o reconhecimento da estrutura e do funcionamento do Centro de Especialidades Odontológicas (CEO) e do setor de odontologia hospitalar de hospitais ligados à IES ${ }^{16}$.

Os ECS contribuem para uma formação coerente com o mundo do traba1 ho ${ }^{17,18}$ principalmente porque possibilita o conhecimento da realidade dos serviços e 
da comunidade. Um aspecto a ser considerado é que o planejamento das ações deve ocorrer com bases nessas necessidades, além de oportunizar a vivência do trabalho em equipe ${ }^{13,17}$.

A inserção dos alunos no cotidiano dos serviços desperta-os para a atuação futura na saúde pública ${ }^{18}$, dado também revelado em estudo avaliativo sobre o PET-Saúde ${ }^{7-9}$.

A figura do profissional dos serviços aparece como importante mediador do processo de ensinoaprendizagem $^{15,17}$. Além disso, a presença de alunos foi vista como uma motivação para esses profissionais no sentido de incentivá-los a buscar e atualizar conhecimentos $^{19}$. Um dos trabalhos dedica um tópico específico para abordar a educação permanente e relata o desenvolvimento de oficinas com os profissionais do serviço, baseadas na problematização do cotidiano de trabalho, o que permitiu a construção e reconstrução reflexiva das práticas em saúde, com o posterior reconhecimento da efetividade no processo de trabalho pelos profissionais que participaram ${ }^{15}$. A comunidade também aparece como um importante componente na efetividade da aprendizagem $^{17}$.

A garantia da interdisciplinaridade como característica indispensável dos ECS foi recorrente em vários trabalhos ${ }^{13,17,20,21}$. Destaca-se o relato da formação de equipes de alunos de diferentes cursos da área da saúde que eram inseridos conjuntamente nas $\mathrm{UBS}^{20}$.

$\mathrm{Na}$ busca desses elementos que qualificam os estágios, indiscutivelmente, a participação dos alunos no PET-Saúde contribuiu para a formação profissional pautada nos princípios da cidadania, no reconhecimento da autonomia dos usuários, na interação com a população e com as equipes de saúde, na busca de soluções para os problemas identificados e no envolvimento com os resultados da assistência ${ }^{22}$.

Como principais limites, os trabalhos analisados apontam a visão dos gestores sobre a formação no serviço, a alta demanda populacional aliada à baixa resolutividade das ações odontológicas ${ }^{19} \mathrm{e}$ a infraestrutura dos campos de estágio que, nem sempre é adequada para receber os alunos ${ }^{18,19}$.

\section{Sobre a construção de um modelo lógico}

De posse desses dados e sustentados pelo referencial teórico que apoiou o delineamento do estudo, sumarizado na Figura 1, elaborou-se o modelo lógico-ideal de ECS como ilustrado na Figura 2.

O modelo ressalta a importância de se manter uma inserção longitudinal dos estudantes nos cenários de produção de saúde, de modo a oferecer a eles a possibilidade de experienciar uma clínica ampliada em Atenção Primária, propiciando o entendimento da cavidade bucal de maneira articulada ao indivíduo em suas dimensões biopsicossociais.

O modelo prioriza a compreensão da organização dos serviços de saúde de forma integral e o entendimento do SUS no seu cotidiano, com seus limites e desafios, permitindo o rompimento de conceitos pré-estabelecidos e a apropriação do conhecimento real.

Pedagogicamente, apoia-se na problematização e conta com a colaboração de profissionais dos serviços de saúde como mediadores pedagógicos. 


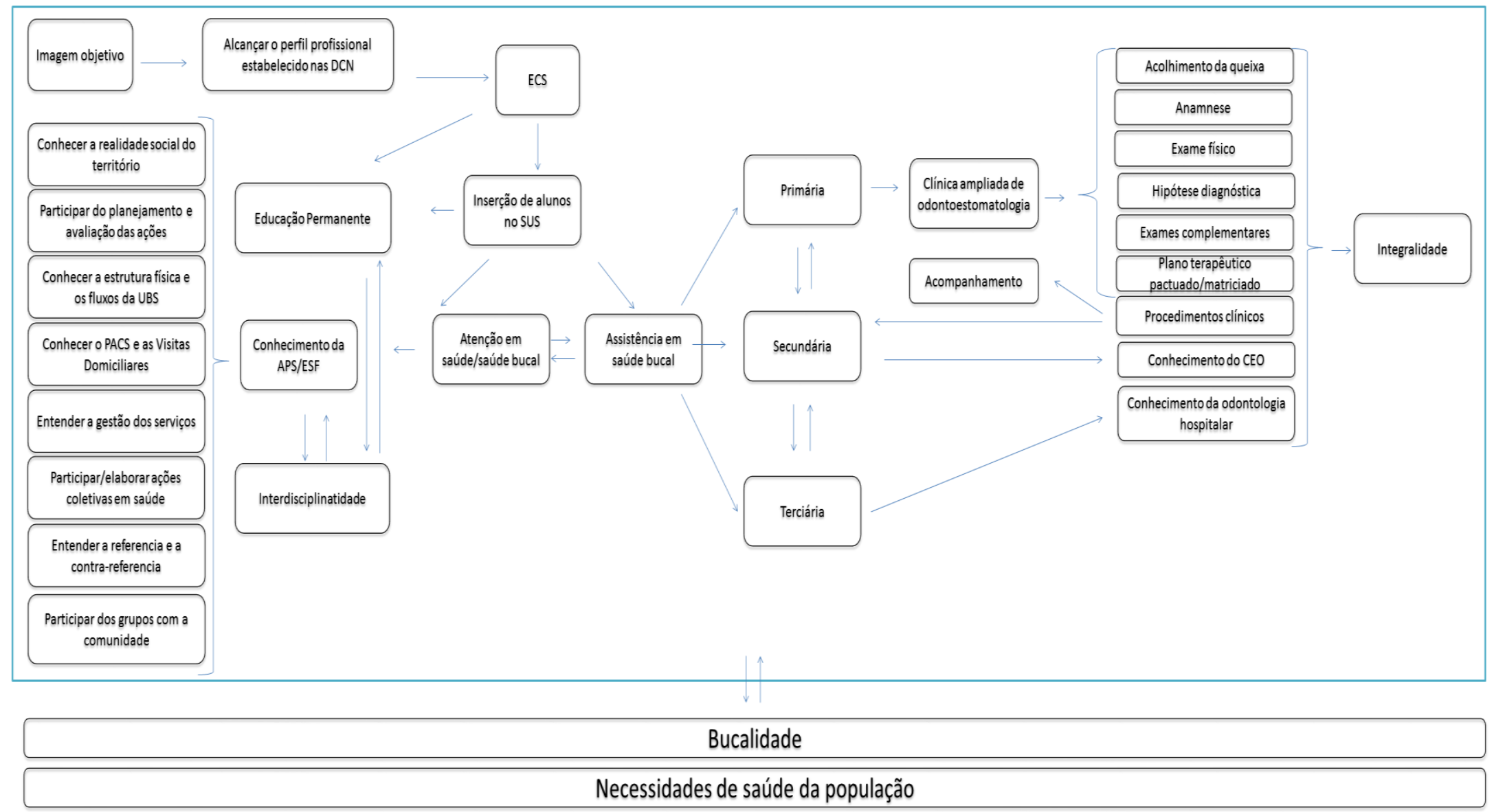

Figura 2: Modelo lógico-ideal de Estágio Curricular Supervisionado (ECS) 


\section{CONSIDERAÇÕES FINAIS}

Após a publicação das DCN, a ABENO se propôs a capitanear e subsidiar processos de reestruturação curricular nas IES e estabeleceu, a partir de ampla discussão com coordenadores e professores, diretrizes norteadoras, particularmente para os estágios supervisionados ${ }^{6}$. Tendo em vista o propósito de produção de reflexão e análise crítica dos assuntos pertinentes à educação superior na Revista da referida Associação, esse estudo se limitou a este único periódico, ao entender que representava um dos principais veículos de comunicação e divulgação de experiências nessa temática. Novas buscas são necessárias para adensar a discussão acerca do modelo lógico proposto.

Os estágios supervisionados, emquanto tema de publicação, ganham corpo após uma década das publicações das DCN e das diretrizes norteadoras dos estágios propostas pela ABENO, o que confirma se tratar de um processo gradual, conformado de forma heterogênea pelo território brasileiro. Cada IES se organiza de acordo com suas possibilidades, crenças e ideologias, com disparidades em relação aos locais de realização, duração e atividades desenvolvidas.

Quanto às contribuições para a formação do egresso, percebeu-se que o profissional do serviço tem uma função primordial nessa relação, mas estudos qualitativos são necessários para que seja possível revelar de que forma tocam os estudantes e como a experiência em serviço se materializa em transformação favorável ao perfil de profissional almejado.

A proposta de um modelo lógico visou contribuir na reflexão e elaboração de propostas para os ECS. Confeccionado com base nos trabalhos analisados, no referencial teórico e na experiência do Programa de Educação pelo Trabalho para a Saúde, demonstra que deve ser oferecido aos estudantes a possibilidade de inserirse na rotina dos serviços de saúde, o que poderia viabilizar a compreensão do SUS em seu contexto real e, por conseguinte, as minúcias da organização dos serviços de saúde bucal.

O modelo proposto, entretanto, requer o desenvolvimento de estudos que permitam identificar os limites e a melhor forma de viabilizá-lo.

\section{ABSTRACT \\ Ideal-logical model for supervised curricular training: education for working in dental education}

The study sought to develop a logical model of Supervised Curricular Training for undergraduate courses in dentistry. This transversal study raised all abstracts and articles in Brazilian Association of Dental Education journals published between 2003 and 2012. Were included in the study 23 papers containing the word 'stage' in their titles. The variables studied were year of publication, place of performance, duration, developed activities, contributions to the formation, role of professional services and limits found. The data relating to the first three variables were entered in a spreadsheet in Microsoft $®$ Office Excel 2007 and analyzed using frequency distribution. The other variables were analyzed using a qualitative method. Most work has been published in the years $2012(13.04 \%)$, $2011(17.39 \%)$ and 2010 (17.39\%). Much of the stages were performed with in the Unified Health System (SUS) (73.91\%) being the most developed, strictly, the Primary Health Care (39.13\%). Most 
intern ships lasted one semester $(69.56 \%)$. The Supervised Curricular Training contributes to a formation coherent with the world of work, favors the experience of teamwork and opens students for future action in public health. The proposed logic model highlights the importance of longitudinal integration in health services, prioritizes understanding the organization of health services in a comprehensive manner and understanding of the SUS in their daily lives, with its limitations and challenges.

Descriptors: Dentistry. Education, Higher. Human Resources. Oral Health.

\section{REFERÊNCIAS}

1. Brasil. Casa Civil. Lei 11.788 , de 25 de setembro de 2008. Dispõe sobre o estágio de estudantes; altera a redação do art. 428 da Consolidação das Leis do Trabalho - CLT, aprovada pelo Decreto-Lei no 5.452, de 1o de maio de 1943, e a Lei no 9.394, de 20 de dezembro de 1996; revoga as Leis nos 6.494, de 7 de dezembro de 1977, e 8.859, de 23 de março de 1994, o parágrafo único do art. 82 da Lei no 9.394, de 20 de dezembro de 1996, e o art. 6o da Medida Provisória no 2.164-41, de 24 de agosto de 2001; e dá outras providências. Disponível em: http://www.planalto.gov.br/ccivil_03/ ato2007-2010/2008/lei/111788.htm.

Acesso em: 30 de abril de 2013.

2. Burgatti JCB. A contribuição do estágio curricular supervisionado no desenvolvimento da dimensão ética da competência de graduandos em Enfermagem [tese]. São Paulo: Escola de Enfermagem, Universidade de São Paulo. São Paulo, 2012.
3. Werneck MAF, Senna MIB, Drumond MM, Lucas SD. Nem tudo é estágio: contribuições para o debate. Cien Saude Col. 2010;15(1):221-31.

4. Feuerwerker LCM, Capozzolo AA. Mudanças na formação dos profissionais de saúde: alguns referenciais de partida do eixo Trabalho em Saúde. In: Capozzolo AA, Casseto SJ, Henz AO (org). Clínica Comum: itinerários de uma formação em saúde. São Paulo: Hucitec, 2013.

5. Brasil. Ministério da Educação. Conselho Nacional de Educação. Câmara de Educação Superior. Resolução CNE/CES No 3, de 19 de fevereiro de 2002. Institui as Diretrizes Curriculares Nacionais do Curso de Graduação em Odontologia. Brasília, 2002. Disponível em: http://portal.mec.gov.br/cne/arquivos/ pdf/CES032002.pdf.

6. Associação Brasileira de Ensino Odontológico (ABENO). Diretrizes da ABENO para a definição do estágio supervisionado nos cursos de Odontologia. Rev ABENO, 2(1):13-8, 2002.

7. Fonsêca, GS, Junqueira SR. Programa de Educação pelo Trabalho para a Saúde: ressignificando a formação dos profissionais de saúde. Curitiba: Editora Appris, 2014.

8. Fonsêca GS, Junqueira SR. Programa de Educação pelo Trabalho para a Saúde da Universidade de São Paulo (Campus Capital): o olhar dos tutores. Cien Saude Col. 2014; 19:1151-62.

9. Fonsêca, GS, Junqueira SR, Zilbovicius C, Araujo ME. Education through work: reshaping the 
education of health professionals. Interface (Botucatu) 2014;50: 571-83.

10. Medina MG, Silva GAP, Aquino R, Hartz ZMVS. Uso de modelos teóricos na avaliação em saúde: aspectos conceituais e operacionais. In: Hartz ZMA, Silva LMVS, editoras. Avaliação em Saúde: dos Modelos Teóricos à Prática na Avaliação de Programas e Sistemas de Saúde. Salvador: EDUFBA; Rio de Janeiro: Fiocruz; 2010. p. 41-63.

11. Bardin L. Análise de Conteúdo. São Paulo: Edições 70; 2011. 276 p.

12. Leme PAT, Meneghim MC, Mialhe F. Estágio extramuro na unidade de Saúde da Família - o que pensam os graduandos? Rev ABENO. 2012; 12(1):99.

13. Schubert EW, Schramm CA, Odebrecht CMR, Vizzoto D, Schroeder MDS, Wilhelmsem NCVG. Disciplina de estágio extramuros - relevância para acadêmicos e comunidades. Rev ABENO. 2011;11(2):123.

14. Vieira JMR, Alves-Filho AO, Humberto JSM, Domingues JEG, Padilha NRR, Medina PO, Rebelo MAB. Experiências do Estágio Curricular I: uma aproximação da realidade local. Rev ABENO. 2011;11(2):122

15. Warmling CM, Rossoni E, Hugo FN, Toassi RFC, Lemos VA, Slavutzki SMB, Bercht S, Nunes AA, Rosa AR. Estágios curriculares no SUS: experiências da Faculdade de Odontologia da UFRGS. Rev ABENO. 2011;11(2):63-70.

16. Hugo FN. A Construção do Estágio II do novo currículo do Curso de
Odontologia da UFRGS. Rev ABENO. 2010;10(1):62.

17. Forte FDS, Pessoa TRRF, Freitas CHSM, Carvalho-Junior PMC. Atenção primária na odontologia: importância dos estágios supervisionados. Rev ABENO. 2012;12(1):47.

18. Lobo AEA, Menezes LMB, Cidrak ML. Estágios curriculares sob a ótica do egresso do Curso de odontologia da universidade Federal do Ceará. Rev ABENO. 2012;12(1):10.

19. Lemos VMA, Slavutzky SMB, Rossoni E, Toassi. Estágio curricular em atenção básica da FO/UFRGS: desafios e perspectivas. Rev ABENO. 2010;10(1):57.

20. Lucas RSCC, Medeiros CLSG, Diniz DN, Granville-Garcia AF. Percepção discente dos estágios supervisionados na Odontologia - UEPB: frutos colhidos. Rev ABENO. 2011; 11(2):149.

21. Lemos VMA, Slavutzky SMB, Hugo FN, Davoglio RS, Toassi RFC, Martins AB. Do antigo ao novo currículo: os estágios da FO-UFRGS. Rev ABENO. 2009;9(2):117.

22. Junqueira SR. Integração EnsinoServiço: proposta de aplicação desta parceria para a educação superior. [Tese]. São Paulo: Universidade de São Paulo, Faculdade de Odontologia; 2013.

Correspondência para:

Graciela Soares Fonsêca

e-mail: gracielafonseca@usp.br

Faculdade de Odontologia da Universidade de São Paulo (FOUSP)

Av. Prof. Lineu Prestes, 2227

05558-000 Cidade Universitária - São

Paulo/SP 